\title{
IMEX WENO Schemes for Two-phase Flow Vertical Equilibrium Processes in a Homogeneous Porous Medium
}

\author{
R. Donat, F. Guerrero* and P. Mulet \\ Department of Applied Mathematics. Universitat de Valencia, Av. Dr. Moliner, 50, 46100 Burjassot-Valencia, Spain
}

Received: 18 Jan. 2013, Revised: 21 May. 2013, Accepted: 22 May. 2013

Published online: 1 Sep. 2013

\begin{abstract}
The motion of two-phase flow in a porous medium under the condition of vertical equilibrium can be described by a viscous conservation law that involves a non-convex flux function with two inflection points. In [5], a first order Godunov scheme was used to numerically approximate solutions of the model. In this paper we show that using instead the high resolution Weighted Essentially Non Oscillatory (WENO) technology, and an IMEX strategy to handle the capillary term by an implicit discretization, leads to a noticeable increase in resolution power and efficiency. We carefully discuss the implementation of WENO schemes for the model equation, paying special attention to the choice of the definition of the numerical viscosity. We also present numerical simulations when the capillary number is negligible (i.e., the model is a homogeneous conservation law) and non-negligible (i.e. the model equation becomes a 'viscous' conservation law). The numerical results are compared with those obtained with the method proposed in [5] in terms of accuracy, resolution power and global efficiency.
\end{abstract}

Keywords: Numerical methods, WENO schemes, IMEX schemes, Porous media, Two-phase flow, Vertical equilibrium, Conservation laws

\section{Introduction}

In a porous medium, vertical equilibrium for two-phase flow occurs when the sum of the velocities of the two phases is zero, which amounts to assuming that the motion of two immiscible fluids is governed by the balance between capillary and gravitational forces. Then, the denser fluid flows downward while the lighter fluid flows upward, with velocities that are equal in magnitude. The assumption of vertical equilibrium is used in the modeling of several two-phase flow problems of engineering interest, such as oil and gas reservoirs [1] [2], ground water contamination [3], $\mathrm{CO}_{2}$ storage sites [4], etc.

In [5], a mathematical model for the displacement of a gas phase through a liquid phase in a porous medium is derived from basic principles. Using Darcy's law [6] and mass conservation, the authors obtain a model equation for vertical equilibrium displacements, which in dimensionless variables takes the form

$$
\frac{\partial s}{\partial t}+\frac{\partial}{\partial x} F(s)+N \frac{\partial}{\partial x}\left(F(s) \frac{\partial P_{c}(s)}{\partial x}\right)=0,
$$

where $s(x, t)$ is the liquid phase saturation, $N$ is the capillary number, and $P_{c}(s)$ and $F(s)$ are known functions (see [5] for details). A related model has been proposed in [4] to study the vertical migration of $\mathrm{CO}_{2}$ through a column with periodical layers of different porosity.

The second and third terms in (1) represent the contributions of buoyancy and capillary effects, respectively. The flux function $F(s)$ in the buoyancy term depends on the absolute and relative permeabilities between the two phases and the porous media, and turns out to be a bell-shaped function, with a local maximum and two inflection points. As pointed out in [5], the fact that $F(s)$ changes concavity twice adds more difficulties to the mathematical and numerical analysis of the associated models than the simpler (and rather extensively

\footnotetext{
*Corresponding author e-mail: guecor@uv.es
} 
studied) Buckley-Leverett model, for which the flux is an $S$-shaped function, with only one inflection point.

The specific form of $F(s)$ depends on the model used for the relative permeabilities. In [5], the Brooks-Corey models [7] were considered, leading to the following expression for the flux function

$$
F(s)=\frac{s^{2}(1-s)^{2}}{s^{2}+(1-s)^{2}} .
$$

The third term in (1) is due to capillary effects and it involves the capillary pressure, as a function of the liquid saturation $s$. As in [5], we shall consider the following expression for the normalized capillary pressure $P_{c}(s)$,

$$
P_{c}(s)=s^{-1 / 2},
$$

which is consistent with Brooks-Corey models and Leverett relationship [8]. This term approaches zero when the capillary number $N$ is small, which occurs when the size of the pore of the medium is very large. Capillary effects are, thus, negligible in many situations of practical interest and, in such cases, (1) reduces to a scalar conservation law, with a non-convex flux.

In the mathematical literature, the standard approach to obtain reliable approximations to the solution of hyperbolic conservation laws and systems is given by the so-called conservative approach. Numerical solutions obtained with conservative schemes represent discontinuities in the true solution of a homogeneous conservation law as sharp transitions, involving often only a few discrete solution values, and are often called 'shock capturing' schemes. Many of these conservative schemes follow the path laid out by Godunov and use the solution of the so-called Riemann Problem, for which the initial data is a step function, as a building block of the scheme. We refer the interested reader to [9] for details on Godunov schemes.

The conservative framework is only recently finding its place in the engineering literature, where the method of characteristics was often used to handle hyperbolic equations, probably due to the physical intuition behind it. For the model problem (1), a Godunov scheme is proposed in [5], and its behavior is tested against a known exact solution of a specific initial-boundary value problem for (1) when $N=0$. However, one of the main advantages of following the 'conservative approach' in the numerical simulation of equations such as (1) is the fact that there exists nowadays a (rather large) pool of well established high resolution shock capturing numerical techniques. Understanding the principles behind the basic design of these numerical techniques allows the user to adapt the technique to the particular problem at hand, in order to obtain well resolved and robust numerical simulations.

In this paper, we discuss the implementation of the modern Weighted Essentially Non-Oscillatory (WENO) schemes for the model equation (1). As we shall see, the general setting for WENO schemes requires an estimate of the appropriate numerical viscosity, which depends on the flux function $F(s)$. The computation of this estimate for a bell-shaped non-convex flux, such as (2) only depends on the knowledge of the two inflection points. To define properly the numerical viscosity is of paramount importance. Otherwise, the scheme may give rise to unwanted oscillations.

We propose then numerical schemes for the $N=0$ and $N \neq 0$ cases that incorporate the WENO construction in the numerical fluxes, instead of the lower order Godunov construction used, for example, in [5], [4] or [10]. We propose implicit-explicit (IMEX) Runge-Kutta solvers to overcome the stability restrictions on the time step size when $N \neq 0$. The nonlinear systems that arise in this formulation are solved by a lagged diffusivity fixed point solver. The results obtained are compared with those in [5] from the point of view of the accuracy, resolution power and global efficiency.

The paper is organized as follows. In section 2, we recall the basic ingredients of the WENO schemes that we propose for the discretization of the buoyancy term, paying special attention to the numerical viscosity of the scheme for non-convex fluxes. In addition, the IMEX strategy that we use to handle the capillary term is shown. In section 3 , we show the numerical results obtained for a set of numerical experiments for the cases $N=0$ and $N \neq 0$, together with a comparison of computational times and errors with respect to the Godunov type scheme of [5]. Finally, in section 4 we present some conclusions and perspectives for future work.

\section{WENO schemes for the model equation}

Shock-capturing techniques for the numerical approximation of discontinuous solutions to hyperbolic conservation laws are based on a classical result by Lax and Wendroff (see e.g. [9]) that establishes that if the limit solution of a conservative scheme has discontinuities, these will propagate according to the Rankine-Hugoniot conditions. This result ensures that conservative schemes capture the discontinuities in the solution, and gives rise to the shock-capturing terminology.

Shock capturing schemes are now routinely used in applications involving discontinuous solutions of hyperbolic conservation laws. In [5] a first order Godunov scheme is proposed in order to obtain numerical simulations of the model problem without capillary effects. As we shall demonstrate, the use of more sophisticated techniques, such as the WENO construction advocated in this paper, can lead to a noticeable increase in resolution and efficiency, providing a reliable tool to be used in more general situations.

The discrete set-up we shall consider is as follows: a uniform grid is laid out on the spatial domain, taken as $[0,1]$ for simplicity. For convenience, the grid-points are $x_{j}=\left(j+\frac{1}{2}\right) h, j=0,1, \ldots m-1, m \cdot h=1$, and $\left[x_{j-\frac{1}{2}}, x_{j+\frac{1}{2}}\right]$ 
is the $j$-th computational cell, $j=0,1, \ldots, m-1$, where the cell-endpoints are $x_{j+\frac{1}{2}}=(j+1) h, j=-1, \ldots, m-$ 1. Notice that, with this definition, the domain boundaries $x=0$ and $x=1$ correspond to cell-endpoints. The time interval $[0, T]$ is uniformly discretized, hence $t_{n}=n \cdot k, k$, where $k$ is the discrete time-step and $n_{T} k=T$ for some integer $n_{T}$. It is well known (see e.g. [9]) that $k$ and $h$ must be related by a stability condition, which depends on the type of equation being discretized, as well as the type of numerical technique used.

Any conservative scheme computes discrete values, $s_{j}^{n}$, associated to the discrete grid-points $\left(x_{j}, t_{n}\right)$. In finite-volume schemes, such as the Godunov scheme proposed in [5], these discrete values are interpreted as approximations to the cell-averages of the true solution $s(x, t)$, while in finite-difference conservative schemes, such as the WENO scheme advocated in this paper, $s_{j}^{n} \approx s\left(x_{j}, t_{n}\right)$, i.e. they are considered as approximations to the point-values of the true solution.

WENO schemes are nowadays routinely used for applications involving convex conservation laws and systems. We shall see next that its application in the non-convex case only requires a careful definition of the numerical viscosity of the scheme.

\section{1 $N=0:$ The homogeneous case}

According to [5], a fully discrete conservative numerical scheme for a homogeneous conservation law, such as the model equation (1) when $N=0$, has the form

$$
s_{j}^{n+1}=s_{j}^{n}-\frac{k}{h}\left[\hat{F}_{j+1 / 2}^{n}-\hat{F}_{j-1 / 2}^{n}\right],
$$

where $\hat{F}_{j+1 / 2}^{n}$ is the numerical flux through the boundary of the cell. In any conservative scheme, this numerical flux is computed using a set of values around the interface $x_{j+1 / 2}$. For a first order scheme, only the nearest neighbors, $s_{j}^{n}$ and $s_{j+1}^{n}$ are involved, but in higher order schemes more values might be necessary in order to improve the accuracy of the scheme. Hence, in general, $\hat{F}_{j+1 / 2}^{n}=\hat{F}\left(s_{j-r}^{n}, \ldots, s_{j+r+1}^{n}\right)$, where $r \geq 0$ and $\hat{F}$ is known as the numerical flux function, the trademark of the scheme, which determines many of its properties.

In Godunov's method, the design of the numerical flux function is based on the solution of a Riemann problem at each cell-boundary $x_{j+1 / 2}$, hence $F_{j+1 / 2}^{n}=\hat{F}\left(s_{j}^{n}, s_{j+1}^{n}\right)$. In the WENO schemes designed in [12] (see also $[15,16]$ and references therein) the numerical flux function is based on the entropy-satisfying Local-Lax-Friedrichs scheme, for which the numerical flux function at an interface separating two states, $s_{l}$ and $s_{r}$, is given by

$$
\hat{F}^{\mathrm{LLF}}\left(s_{l}, s_{r}\right)=\frac{1}{2}\left(F\left(s_{l}\right)+F\left(s_{r}\right)-\alpha\left(s_{r}-s_{l}\right)\right),
$$

where the parameter $\alpha$ above should satisfy

$$
\alpha \geq \max \left\{\left|F^{\prime}(s)\right|, s \in\left[s_{l}, s_{r}\right]\right\}
$$

where $\left[s_{l}, s_{r}\right]$ represents the closed interval $s_{l} \leq s \leq s_{r}$ if $s_{l} \leq s_{r}$ or $s_{r} \leq s \leq s_{l}$ if $s_{r} \leq s_{l}$. The reason for this requirement can be understood by noticing that

$$
\hat{F}^{\mathrm{LLF}}\left(s_{l}, s_{r}\right)=F^{-}\left(s_{r}\right)+F^{+}\left(s_{l}\right)
$$

with

$$
F^{-}(s)=\frac{1}{2}(F(s)-\alpha s), \quad F^{+}(s)=\frac{1}{2}(F(s)+\alpha s)
$$

The choice of $\alpha$ specified in (6) guarantees that the signed fluxes $F^{ \pm}$satisfy $\left(F^{+}\right)^{\prime} \geq 0,\left(F^{-}\right)^{\prime} \leq 0$ in $\left[s_{l}, s_{r}\right]$, i.e. around the interface. Hence, $F^{ \pm}$in (7) are evaluated at the upwind point, which turns out to be crucial for the stability of the conservative scheme having (5) as numerical flux.

If $F(s)$ is a convex (or concave) function, then

$$
\alpha=\max \left\{\left|F^{\prime}(s)\right|, s \in\left[s_{l}, s_{r}\right]\right\}=\max \left\{\left|F^{\prime}\left(s_{l}\right)\right|,\left|F^{\prime}\left(s_{r}\right)\right|\right\}
$$

satisfies (6).

However, for a bell-shaped, non-convex, function, such as (2), the value of $\alpha$ depends on the location of the two inflection points $S_{1}, S_{2}$ with respect to the values $s_{l}, s_{r}$. In this case, an appropriate definition is as follows

$$
\alpha=\max \left\{\left|F^{\prime}(s)\right| / s \in\left\{s_{l}, s_{r}\right\} \cup\left(\left\{S_{1}, S_{2}\right\} \cap\right] s_{l}, s_{r}[)\right\},
$$

where $] s_{l}, s_{r}$ [ is the open interval determined by $s_{l}$ and $s_{r}$.

In subsection 3.4, we see that to choose the appropriate definition is a key point to obtain a well behaved numerical solution.

The scheme (4) with the numerical flux function at each interface defined by the formula (5), or equivalently,

$$
\begin{aligned}
& \hat{F}_{j+1 / 2}^{n}=F_{j+1 / 2}^{-}\left(s_{j+1}^{n}\right)+F_{j+1 / 2}^{+}\left(s_{j}^{n}\right), \\
& F_{j+1 / 2}^{ \pm}(s)=F(s) \pm \alpha_{j+1 / 2} s
\end{aligned}
$$

where $\alpha_{j+1 / 2}$ is computed as specified before, with $s_{l}=s_{j}^{n}$ and $s_{r}=s_{j+1}^{n}$, gives rise to a first order accurate conservative scheme, since it is easy to prove that (10) leads to a flux difference satisfying

$$
\frac{1}{h}\left(\hat{F}_{j+1 / 2}^{n}-\hat{F}_{j-1 / 2}^{n}\right)=\left.\partial_{x}(F(s))\right|_{\left(x_{j}, t_{n}\right)}+O(h),
$$

for $s_{j}^{n}=s\left(x_{j}, t_{n}\right)$.

Its numerical behavior is similar to that of the Godunov scheme used in [5], but with a larger degree of smearing around the location of the shocks in the numerical solution. 
A high order WENO scheme is designed in such a way that the flux difference is a better approximation of $\left.\partial_{x}(F(s))\right|_{\left(x_{j}, t_{n}\right)}$, thus allowing for an improvement in spatial accuracy. The WENO numerical flux has the form

$$
\begin{aligned}
\hat{F}_{j+1 / 2}^{n}= & \mathscr{R}^{+}\left(F_{j+1 / 2}^{+}\left(s_{j-r}^{n}\right), \ldots, F_{j+1 / 2}^{+}\left(s_{j+r}^{n}\right) ; x_{j+1 / 2}\right)+ \\
& \mathscr{R}^{-}\left(F_{j+1 / 2}^{-}\left(s_{j-r+1}^{n}\right), \ldots, F_{j+1 / 2}^{-}\left(s_{j+r+1}^{n}\right) ; x_{j+1 / 2}\right) .
\end{aligned}
$$

Here $\mathscr{R}^{ \pm} \quad$ represent specific non-oscillatory, upwind-biased, data-dependent polynomial reconstruction procedures, which are evaluated at the cell boundary $x_{j+1 / 2}$. These procedures are nowadays almost a black-box type of routine (see [15] and references therein).

The number of points involved in the WENO flux computation (11) is determined by $r$. This number determines also the spatial accuracy of the flux difference. It can be proven (see [15]) that, under appropriate smoothness conditions,

$$
\frac{1}{h}\left(\hat{F}_{j+1 / 2}^{n}-\hat{F}_{j-1 / 2}^{n}\right)=\left.\partial_{x}(F(s))\right|_{\left(x_{j}, t_{n}\right)}+O\left(h^{2 r+1}\right),
$$

hence the spatial order of accuracy of the associated conservative scheme(4) is $2 r+1$. For the WENO5 [12] construction $r=2$. When $r=0$, we recover (10).

A WENO numerical flux computation amounts, then, to the use of a sophisticated, upwind-biased, non-oscillatory reconstruction technique on the functions $F_{j+1 / 2}^{ \pm}(s)=F(s) \pm \alpha_{j+1 / 2} s$, where $\alpha_{j+1 / 2}$ is a parameter associated to the $x_{j+1 / 2}$ interface, whose value is computed as specified above, with $s_{l}=s_{j}^{n}$ and $s_{j+1}^{n}$. These reconstruction procedures are, however, straightforward to implement, following the steps outlined in [15].

We end this section by recalling (see e.g. [9]) that the stability condition for a numerical scheme such as (4) is of the type

$$
\frac{k}{h} \max \left|F^{\prime}(s)\right| \leq C \leq 1
$$

where the value $C$ depends on the specific method being used.

We propose the following explicit Runge-Kutta 2 scheme to compute the time evolution of eq. (1) when $N=0$ :

$$
\begin{aligned}
& s^{n+1 / 2}=s^{n}+\frac{k}{2} \mathscr{L}^{n} \\
& s^{n+1}=s^{n}+k \mathscr{L}^{n+1 / 2}
\end{aligned}
$$

where

$$
\mathscr{L}_{j}^{l}=\frac{1}{h}\left(\hat{F}_{j+1 / 2}^{l}-\hat{F}_{j-1 / 2}^{l}\right), \quad 1 \leq j \leq m
$$

and $\hat{F}_{j+1 / 2}^{l}$ is the WENO numerical flux.

\section{$2.2 N \neq 0$ : The viscous case}

When capillary pressure is included in the model, eq. (1) becomes a so-called viscous conservation law. The model equation is now of parabolic type, hence its solutions are smooth. However, it is expected that for small values of $N$, the convection processes due to buoyancy lead to very sharp transitions that resemble shocks. In such cases, it is advantageous to still use the high resolution shock capturing numerical flux functions described in the previous section, combined with an appropriate discretization of the parabolic term describing the capillary effects, in order to get a high resolution numerical approximation to the exact solution of the model problem. form

In [5], the authors propose a numerical scheme of the

$$
s_{j}^{n+1}=s_{j}^{n}-\frac{k}{h}\left[\hat{F}_{j+1 / 2}^{n}-\hat{F}_{j-1 / 2}^{n}\right]+B_{j}^{n}
$$

where the conservative discretization of the buoyancy term is based on Godunov's numerical flux, and $B_{j}^{n}$ represents a second order discretization of the capillary term $N \frac{\partial}{\partial x}\left(F(s) \frac{\partial P_{c}(s)}{\partial x}\right)$, in eq. (1).

We propose to use the WENO5 numerical fluxes defined in the previous section $(r=2)$ for the conservative discretization of the buoyancy term, combined with the same second order accurate discretization of the capillary term as in [5].

It is known that the stability requirements of the capillary terms lead to an excessive restriction in the size of the time step $k$. Then, we propose to use an IMEX scheme: implicit for the parabolic term and explicit for the hyperbolic term. In addition, for time discretization, we consider a second order Runge-Kutta scheme.

Decoupling the spatial and temporal issues, we rewrite eq. (1) as follows:

$$
\frac{d s}{d t}=L(s)+C(s)
$$

where $L(s)$ denotes the advective term $-\frac{\partial f(s)}{\partial x}$ and $C(s)=$ $-N \frac{\partial}{\partial x}\left[F(s) P^{\prime}(s) \frac{\partial s}{\partial x}\right]$ is the diffusive capillary term.

We shall consider the following Implicit - Explicit (IMEX) Runge-Kutta 2 scheme described in [17]:

$$
\begin{aligned}
& s^{n+1 / 2}=s^{n}+\frac{k}{2}\left(\mathscr{L}^{n}+\mathscr{C}^{n+1 / 2}\right) \\
& s^{n+1}=s^{n}+k\left(\mathscr{L}^{n+1 / 2}+\mathscr{C}^{n+1}\right)
\end{aligned}
$$

where the term $\mathscr{C}^{l}$ is the vector that contains the second order spatial discretization of the parabolic term $C(s)=-N \frac{\partial}{\partial x}\left[F(s) P^{\prime}(s) \frac{\partial s}{\partial x}\right]$, obtained from the values $s_{j}^{l}$, $j=0, \ldots, m, l=n, n+1 / 2$, as follows: 


$$
\begin{aligned}
& \mathscr{C}_{j}^{l}=\frac{1}{2 h^{2}}\left[\left(g_{j+1}^{l}+g_{j}^{l}\right) s_{j+1}^{l}-\left(g_{j+1}^{l}+2 g_{j}^{l}+g_{j-1}^{l}\right) s_{j}^{l}+\right. \\
& \left.\left(g_{j}^{l}+g_{j-1}^{l}\right) s_{j-1}^{l}\right]
\end{aligned}
$$

where

$$
g_{j}^{l}=-N F\left(s_{j}^{l}\right) P^{\prime}\left(s_{j}^{l}\right),
$$

for $j=0, \ldots, m+1$, and $l=n, n+1 / 2$.

Notice that the discretization of the hyperbolic term is computed in an explicit manner, from the known solution values $s_{0}^{l}, \ldots, s_{m}^{l}, l=n, n+1 / 2$, whereas the discretization of the parabolic term is computed in an implicit manner in both steps of the scheme.

It is well known that the stability restrictions on the time step for the IMEX scheme are less severe than those of the explicit scheme, which makes the IMEX scheme more efficient, in some cases, than the explicit scheme [17].

To solve eq.(17) we need to solve a nonlinear system of equations in each one of the two steps. To do this, we perform a Fixed Point Iteration method.

Let us consider the first step in eq.(17). It can be rewritten in matrix form as follows:

$$
M\left(s^{n+1 / 2}\right) s^{n+1 / 2}=Q\left(s^{n}\right)
$$

where $M\left(s^{l}\right)$ is the $m \times m$ tridiagonal matrix given by:

$$
M\left(s^{l}\right)=\left[\begin{array}{ccccc}
B_{1}^{l} & C_{1}^{l} & 0 & \ldots & 0 \\
A_{2}^{l} & B_{2}^{l} & C_{2}^{l} & \ldots & 0 \\
\ldots & \ldots & \ldots & \ldots & \ldots \\
0 & \ldots & A_{m-1}^{l} & B_{m-1}^{l} & C_{m-1}^{l} \\
0 & \ldots & 0 & A_{m}^{l} & B_{m}^{l}
\end{array}\right]
$$

where

$$
\begin{aligned}
B_{j}^{l} & =1+\frac{k}{4 h^{2}}\left(g_{j+1}^{l}+2 g_{j}^{l}+g_{j-1}^{l}\right) \\
A_{j}^{l} & =-\frac{k}{4 h^{2}}\left(g_{j}^{l}+g_{j-1}^{l}\right) \\
C_{j}^{l} & =-\frac{k}{4 h^{2}}\left(g_{j+1}^{l}+g_{j}^{l}\right)
\end{aligned}
$$

and $Q\left(s^{l}\right)$ is a column vector whose components are:

$$
Q\left(s^{l}\right)_{j}=s_{j}^{l}+\frac{k}{2} \mathscr{L}\left(s^{l}\right)_{j}, \quad \text { for } j=2, \ldots, m-1
$$

and

$$
\begin{aligned}
& Q\left(s^{l}\right)_{1}=s_{1}^{l}+\frac{k}{2} \mathscr{L}\left(s^{l}\right)_{1}+\frac{k}{4 h^{2}}\left(g_{1}^{l}+g_{0}^{l}\right) s_{0}^{l} \\
& Q\left(s^{l}\right)_{m}=s_{m}^{l}+\frac{k}{2} \mathscr{L}\left(s^{l}\right)_{m}+\frac{k}{4 h^{2}}\left(g_{m+1}^{l}+g_{m}^{l}\right) s_{m+1}^{l}
\end{aligned}
$$

Since the vector $s^{n+1 / 2}$ is implicit in eq.(20) we have to approximately solve it by means of an iterative method. We propose to use a lagged diffusivity Fixed Point Iteration whose algorithm is as follows:

Input: approximate solution vector $\mathbf{s}^{n}$ at $t=t_{n}$ as a starting value

$$
\begin{gathered}
\mathbf{v}^{0}=\mathbf{s}^{n} \text { and } k=0 \\
\text { while } e>\text { Tol and } k \leq N_{\text {iter }} \\
\text { solve for } \mathbf{v}^{k+1} \text { the linear system } \\
M\left(\mathbf{v}^{k}\right) \mathbf{v}^{k+1}=Q\left(\mathbf{s}^{n}\right) \\
k=k+1 \\
\quad e=\left\|\mathbf{v}^{k+1}-\mathbf{v}^{k}\right\|_{\infty} \\
\text { endwhile } \\
\mathbf{s}^{n+1 / 2}=\mathbf{v}^{k+1} \\
\text { Output: approximate solution vector } \mathbf{s}^{n+1 / 2} \text { of }
\end{gathered}
$$
the nonlinear system $M\left(\mathbf{s}^{n+1 / 2}\right) \mathbf{s}^{n+1 / 2}=Q\left(\mathbf{s}^{n}\right)$

The second step of the IMEX-RK2 method is solved analogously.

To ensure the stability of the explicit numerical scheme (15) proposed in [5], a von Neumann analysis suggests a stability condition of the form (see Appendix A):

$$
\frac{k}{h} \max _{\mathbf{s}}\left|F^{\prime}(s)\right|+\frac{2 k N}{h^{2}} \max _{s}\left|F(s) P_{c}^{\prime}(s)\right| \leq C_{1} \leq 1
$$

Assuming, $m h=1$, where $m$ is the number of points, the condition for the ratio $k / h$ can be expressed as:

$$
\frac{k}{h} \rho_{1}+\frac{2 k N}{h^{2}} \rho_{2} \leq C_{1} \leq 1
$$

or

$$
\frac{k}{h} \leq \frac{C_{1}}{\rho_{1}+2 \rho_{2} m N} .
$$

where $\rho_{1}$ is the maximum value of $\left|F^{\prime}(s)\right|$ and $\rho_{2}$ denotes the maximum value of $\left|F(s) P_{c}^{\prime}(s)\right|$.

According to this estimate, when the number of points $m$ increases, the value of $k / h$ allowed has to decrease. This means that the parabolic term may dictate the maximum time step for stability in a very stringent manner if $2 \rho_{2} m N$ is not very small compared with $\rho_{1}$.

On the other hand, for IMEX-RK2 the stability condition suggested by the analysis performed in Appendix A is of the form:

$$
\frac{k}{h} \max _{\mathbf{s}}\left|F^{\prime}(s)\right| \leq C_{2} \leq 1
$$

which can be rewritten as:

$$
\frac{k}{h} \leq \frac{C_{2}}{\rho_{1}}
$$

Hence, the ratio $k / h$ does not depend on the number of points $m$ considered.

The constants $C_{1}$ and $C_{2}$ in eqs. (25) and (28), respectively, depend strongly on the ODE solver. We show in Appendix A, that for the case of the Euler scheme, both constants are equal to one. 
However, since they are obtained from a stability analysis of a linearization of (1), the precise value of $C_{1}$ and $C_{2}$ is subject to some empirism that has been a matter of research for the present work.

\section{Numerical results}

To test the proposed numerical schemes, we follow [5] and consider that the gas is initially concentrated near the base $(x=1)$ and the rest of the core is filled with liquid. Thus, the initial condition is a step function of the form

$$
s(x, 0)=\left\{\begin{array}{l}
1 \text { for } 0<x \leq a, \\
\beta \text { for } a \leq x<1 .
\end{array}\right\} .
$$

where $\beta$ represents the initial saturation of water in the lower part of the column of the porous media. On a finite domain, such as the core, vertical equilibrium is simulated by sealing the ends of the core. This implies a zero-flux boundary condition, to be imposed on both ends of the computational domain.

For the uniform discretization stated in section 2.1, the domain boundaries correspond to cell-endpoints: $x=0=$ $x_{-1 / 2}$, and $x=1=x_{m-1 / 2}$. Hence, the zero-flux boundary conditions [18] [19] are particularly simple to implement in this setting, since they become

$$
\hat{F}_{-1 / 2}^{n}=0, \quad \hat{F}_{m-1 / 2}^{n}=0, \quad n=0,1,2 \ldots
$$

\section{1 $N=0:$ The homogeneous case}

When $N=0$, (1) becomes a non-convex scalar conservation law. In an infinite domain, equation (1) together with the initial data (30) constitute a so-called Riemann problem, a self-similar hyperbolic initial value problem with piecewise constant data on both sides of an imaginary membrane. Even though the Riemann problem is not of direct interest in applications, it is relatively simple to find analytic solutions, which can be used for the validation and comparison of numerical methods applied to the model problem. The geometric construction described in [5] serves to compute the exact solution to the initial-boundary value problem corresponding to a given set of parameters $a, \beta$ as a combination of two Riemann solutions.

We consider next the test case $a=0.6, \beta=0.3$, and use the exact solution for validation of the numerical technique. Figure 1 shows that our choice of parameters corresponds to situation II of proposition 1 and $\mathrm{V}$ of proposition 2 in [5]. Hence, denoting the states as $(s, F(s))$, the exact solution consists of a shock wave connecting the point $(1,0)$ (at $x=0$ ) in Figure 1 and the intermediate state $\left(s_{1}, F\left(s_{1}\right)\right)$, a rarefaction wave connecting $\left(s_{1}, F\left(s_{1}\right)\right)$ with $(\beta, F(\beta))$, a shock connecting $(\beta, F(\beta))$ with an intermediate state

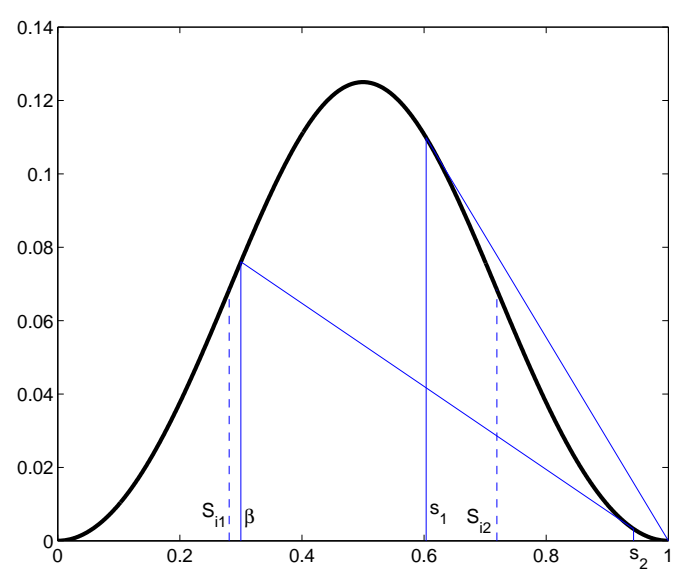

Fig. 1 Graph of the flux function $F(s)$ showing the intermediate states $s_{1}, s_{2}$ and its relationship with $\beta$ and the inflection points $S_{1}, S_{2}$.

$\left(s_{2}, F\left(s_{2}\right)\right)$ and a rarefaction wave between $\left(s_{2}, F\left(s_{2}\right)\right)$ and $(1,0)($ at $x=1)$. The values of $s_{1}$ and $s_{2}$ are obtained as follows (see [5], and Figure 1):

$-\left(s_{1}, F\left(s_{1}\right)\right)$ is such that the tangent line to the graph of

$\mathrm{F}$ at $\left(s_{1}, F\left(s_{1}\right)\right)$ intersects the graph of $\mathrm{F}$ at $(1,0)$.

-The line that connects $(\beta, F(\beta))$ and $\left(s_{2}, F\left(s_{2}\right)\right)$ is tangent to the graph of $\mathrm{F}$ at the point $\left(s_{2}, F\left(s_{2}\right)\right)$.

For $\beta=0.3$ and $F(s)$ given by eq. (1), we have the intermediate states:

$$
\begin{aligned}
& s_{1}=0.6033917422 \\
& s_{2}=0.9429648815
\end{aligned}
$$

The corresponding shock speeds, according to the Rankine-Hugoniot jump condition, can be readily computed as

$$
\begin{gathered}
\sigma\left(1, s_{1}\right)=\frac{F(1)-F\left(s_{1}\right)}{1-s_{1}}=-0.2769531793 \\
\sigma\left(\beta, s_{2}\right)=\frac{F(\beta)-F\left(s_{2}\right)}{\beta-s_{2}}=-0.1132151033
\end{gathered}
$$

The minus sign indicates that shocks are moving to the left in the graphic.

In Figure 2 we show that the numerical scheme correctly reproduces the basic features of the exact solution. Here, the numerical solution is computed on a uniform mesh with $h=.002$ and snapshots at $t=0$, $t=0.3$ and $t=0.5$ are shown. The shock-rarefaction-shock-rarefaction structure of the solution is clearly appreciated, and the shocks are moving to the left, as expected. 


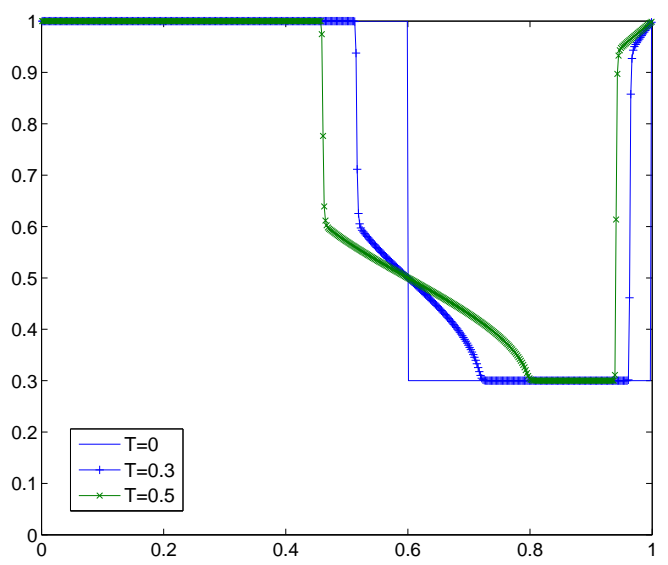

Fig. 2 Numerical solution for the case $N=0$ at $t=0, t=0.3$ and $t=0.5$ (full line, + , and $\mathrm{x}$, respectively) on a uniform mesh with $h=0.002$ and $k / h=0.1$.

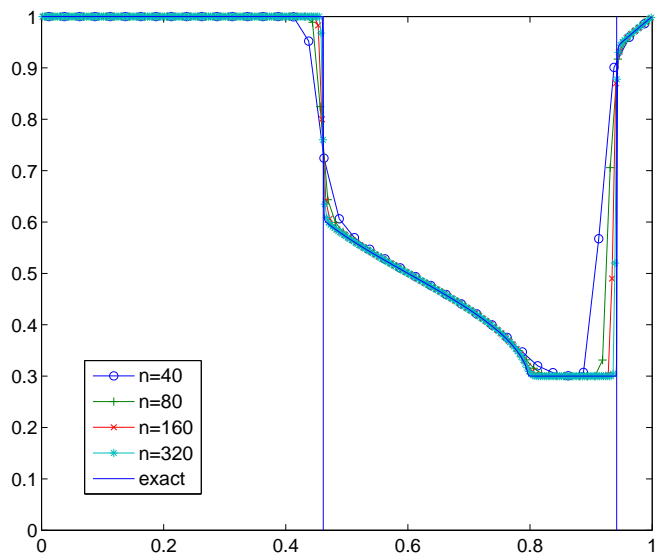

Fig. 3 Numerical solutions corresponding to $h=1 / 40, h=$ $1 / 80, h=1 / 160$ and $h=1 / 320$ at $t=0.5$ with the exact position of the shocks. The solid line corresponds to an 'exact' solution computed on a uniform mesh of 2000 points.

Figure 3 shows the numerical solutions at $t=0.3$ on several meshes, together with the 'true solution', which has also been obtained applying the numerical scheme on a very fine mesh with 2000 points. We also display the exact position of the shocks, marked as vertical lines, computed according to their true Rankine-Hugoniot speed in (33), (34). We clearly observe that decreasing the mesh width leads to finer resolution and better precision in the position of the shocks, which is a clear indication of the

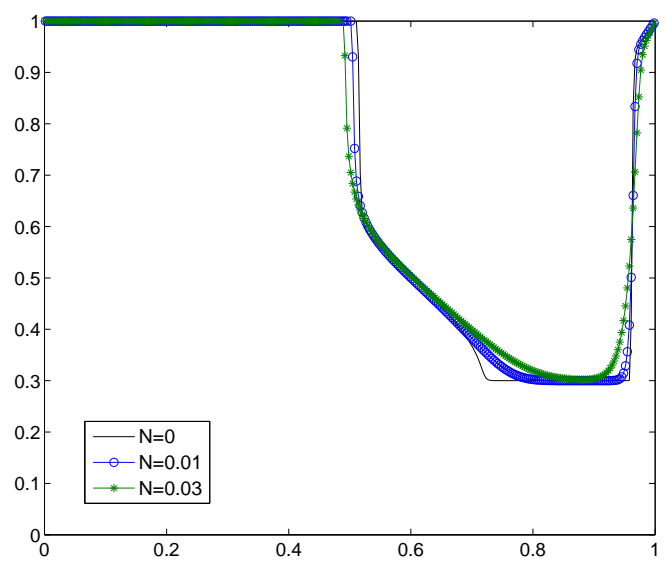

Fig. 4 Comparison between the numerical solutions for $N=0$, $N=0.01$ and $N=0.03$ at $t=0.3$. Here $h=0.002$.

convergence of the numerical scheme. This convergence study shows that the numerical solution converges to the 'true solution' of the problem as the mesh size, $h$, converges to zero. In addition, it shows that the numerical solution is reliable already on coarse meshes. This is a specific feature of high order/high resolution shock capturing schemes, and its relevance can be clearly appreciated in Figure 6, where we compare the numerical results obtained with the WENO5 numerical flux, and the Godunov numerical flux proposed in [5].

We have considered other test cases, using a different set of parameters, obtaining analogous results (see also section 3.3).

\section{$3.2 N \neq 0:$ The viscous case}

As mentioned before, when $N \neq 0,(1)$ becomes a viscous conservation law. For small values of the viscosity parameter $N$, the smooth solution of this parabolic PDE, bears a great similarity to the corresponding solution for $N=0$. The shock discontinuities that occur in the $N=0$ case become smooth transitions when $N \neq 0$, which may be very sharp for very small values of $N$. The use of a conservative formulation for the flux difference leads to an adequate representation of these transitions, even for very small values of $N$. This can be readily appreciated in Figure 4 , where the numerical solution corresponding to (1) with $N=0$ and (30), with $a=0.6$ and $\beta=0.3$, is displayed together with the numerical approximations corresponding to the same initial data and $N=0.01$ and $N=0.03$.

A convergence study is shown in Figure 5. The exact solution is obtained by applying the scheme on an extremely fine mesh of 10240 points. It is worth noticing 


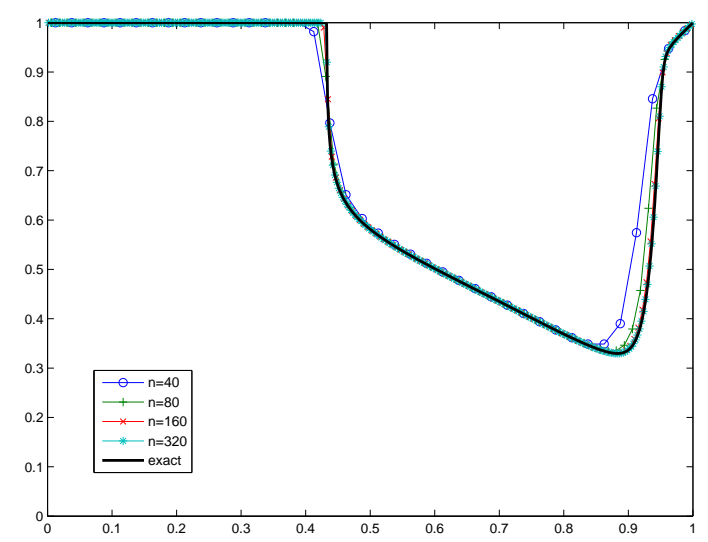

Fig. 5 Convergence study for $N=0.03$ at $t=0.3$

that 'numerical convergence' is attained on rather coarse meshes. This issue will be further considered in the following section.

\subsection{The IMEX-WENO scheme versus the Godunov scheme}

In this section we shall compare the numerical results obtained with our proposed scheme to those obtained with the first order Godunov scheme proposed in [5] for the same model equation.

In Figure 6 we show a comparison of numerical results for the test case $a=0.6, \beta=0.3$ on uniform meshes containing 20 and 40 points on $[0,1]$. We clearly appreciate that, on coarse meshes, the numerical solutions obtained with the IMEX-WENO scheme are more reliable than those obtained with the Godunov scheme. In particular, a highly visible 'dog-leg' effect can be observed in the Godunov simulations around $x=0.6$. This numerical pathology occurs in some schemes with insufficient numerical viscosity; the 'glitch' is proportional to the mesh size, hence it is not observable on the much finer meshes used in the numerical simulations of section 3.1. The bottom row of figure 6 shows snapshots at $t=1$. At this time, the left moving shock that originated at $x=1$ has already interacted with the rarefaction wave and there is no constant state. The glitch at $x=0.6$ still persists on the Godunov simulations, but it does not show up in any of the IMEX-WENO simulations.

In Figure 7 we have chosen a set of parameters that reproduces the situation shown in Figure 3 of [5]. The bottom figure corresponds to the same mesh resolution and can be directly compared with the results shown in [5]. The plots on the top row confirm the observations
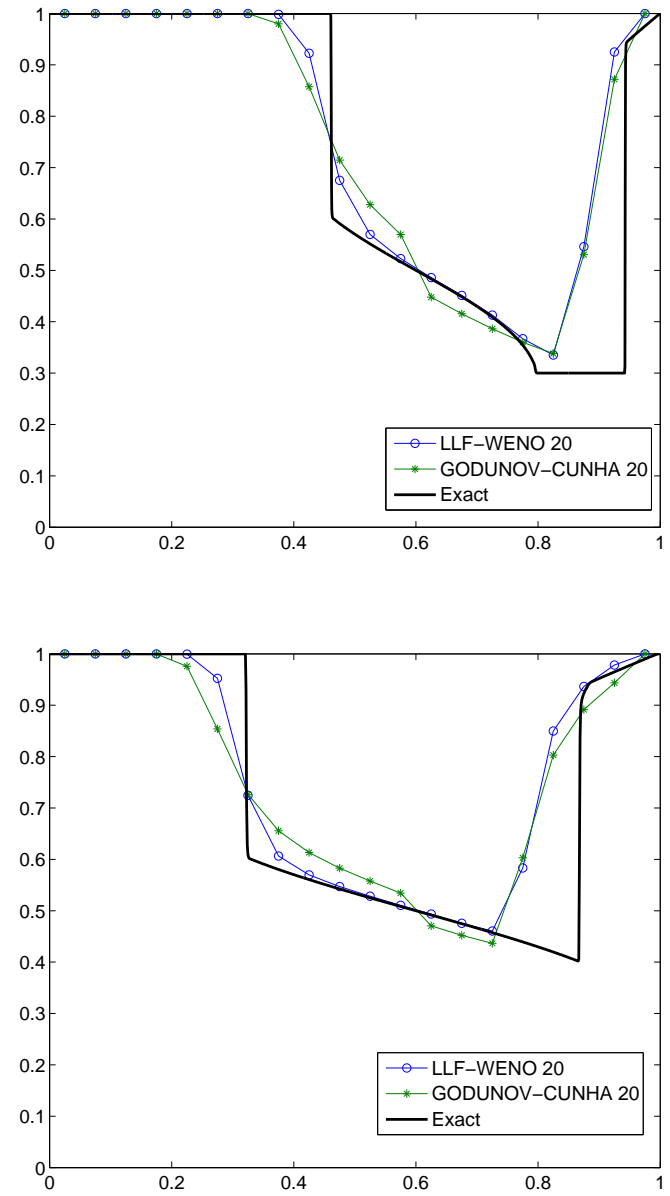

Fig. 6 A comparison of IMEX-WENO versus Godunov numerical simulations on coarse meshes. Top $t=0.3$, bottom $t=1$.

made earlier: in all situations, the IMEX-WENO scheme produces more accurate results. It is worth observing that even on the finest mesh, with 1000 points on $[0,1]$, the first order scheme produces a smeared profile around the location of the second shock, while the IMEX-WENO scheme gives a sharp transition that can be easily identified as a shock.

Figures 8 and 9 show the comparison between the methods for the case $N=0.03, a=0.6, \beta=0.3$ on a uniform mesh with 320 points, together with the 'true' solution, obtained on a very refined mesh. In the zoomed pictures we can observe that the numerical solution obtained with the IMEX-WENO scheme is virtually 'on top' of the true solution, but the solution obtained with the Godunov scheme of [5] is not.

Table 3.3 and Figure 10 show the $L_{1}$-errors (mean errors) of both schemes with respect to the reference 

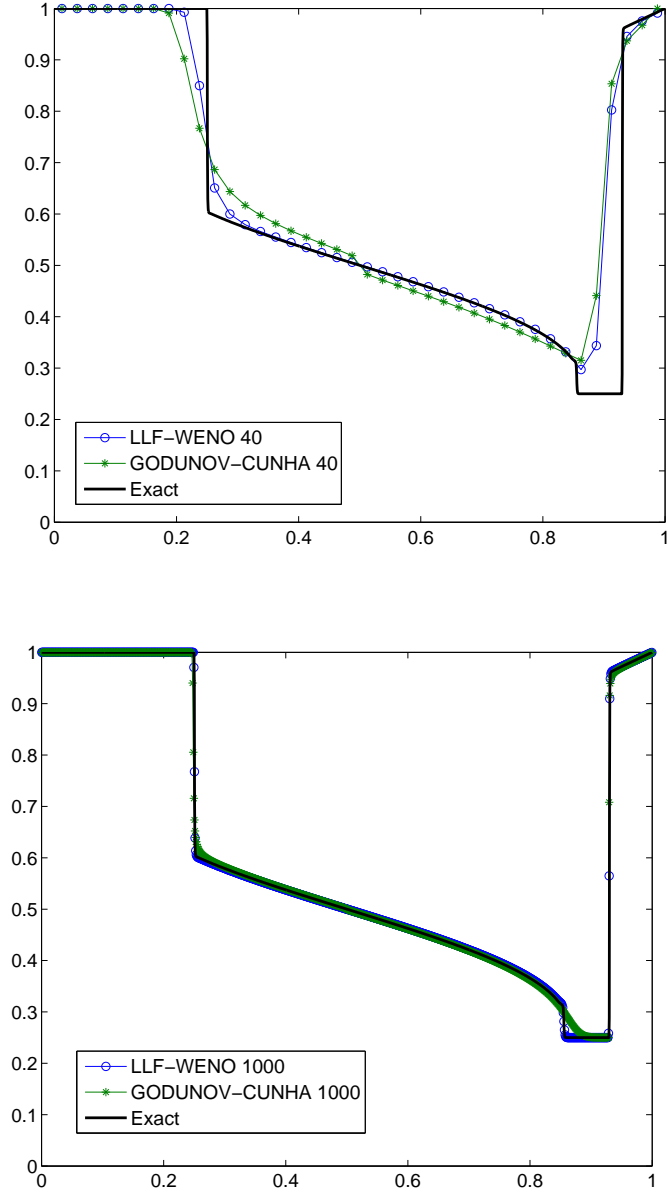

Fig. 7 A comparison of IMEX-WENO versus Godunov numerical simulations. $a=0.5, \beta=0.25, t=0.9$. Top: $h=1 / 40$. Bottom $h=1 / 1000$.

solution, together with the running time of the simulation. In the table, we clearly observe that the mean error decreases with the mesh size, and that the IMEX-WENO simulation is always more reliable (less mean error) than the Godunov simulation.

The data in Table 3.3 also indicate that the IMEX-WENO scheme is more efficient than the Godunov scheme: For the same target error, the IMEX-WENO scheme needs less computation time than the Godunov scheme. The same results are shown graphically in Figure 10, where we clearly appreciate that the data for the IMEX-WENO scheme is always below that of the Godunov scheme.

The values of the errors have been calculated comparing the numerical solutions obtained for each number of points $m$ with a reference solution. This

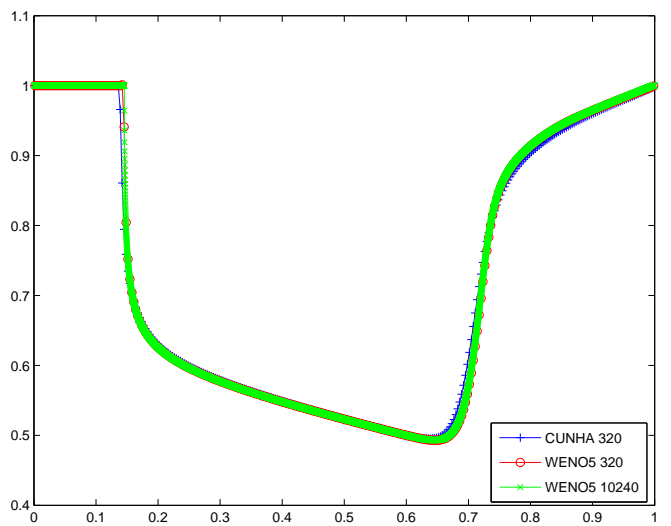

Fig. 8 Comparison between the numerical solutions for our method, Godunov type method used in [5] and the reference solution. The curves are obtained for $T=1.5$ with $N=0.03$, $h=1 / 320$ and $\Delta t / \Delta x^{2}=40$

Table 1 Errors and computation times for the Godunov scheme used in [5] and the IMEX RK2 WENO5 scheme proposed. $N=$ $0.03, \mathrm{t}=1.0$

\begin{tabular}{|c|c|c|c|c|}
\hline & \multicolumn{2}{|c|}{ Godunov } & \multicolumn{2}{c|}{ IMEX-WENO } \\
\hline$m$ & Error & Time (s) & Error & Time (s) \\
\hline 160 & 0.0111 & 0.010 & 0.00527 & 0.015 \\
\hline 320 & 0.00643 & 0.025 & 0.00252 & 0.055 \\
\hline 640 & 0.00363 & 0.140 & 0.00115 & 0.220 \\
\hline 1280 & 0.00205 & 0.940 & 0.000534 & 0.905 \\
\hline 2560 & 0.00118 & 7.505 & 0.000214 & 3.620 \\
\hline
\end{tabular}

reference solution has been obtained by means of the IMEX-WENO scheme with 10240 points.

To obtain the computation times for the Godunov scheme, we have used the stability restriction given by (27).

$$
\frac{k}{h} \leq \frac{C_{1}}{\rho_{1}+2 \rho_{2} m N}
$$

Experimentally, we have checked that this condition is valid for our test problem with $C_{1}=0.89$. In addition, for our flux and pressure functions, eqs. (2) and (3), respectively, it is easy to see that $\rho_{1}=0.3977$ and $\rho_{2}=0.2316$. Therefore, when (35) is not satisfied, oscillations appear in the numerical solution.

On the other hand, the stability condition for the IMEX-WENO scheme given by (29) is valid with $C_{2}=1$.

$$
\frac{k}{h} \leq \frac{C_{2}}{\rho_{1}} .
$$

Accordingly, in this case we have used a constant value for $k / h$ of 2.5 . 

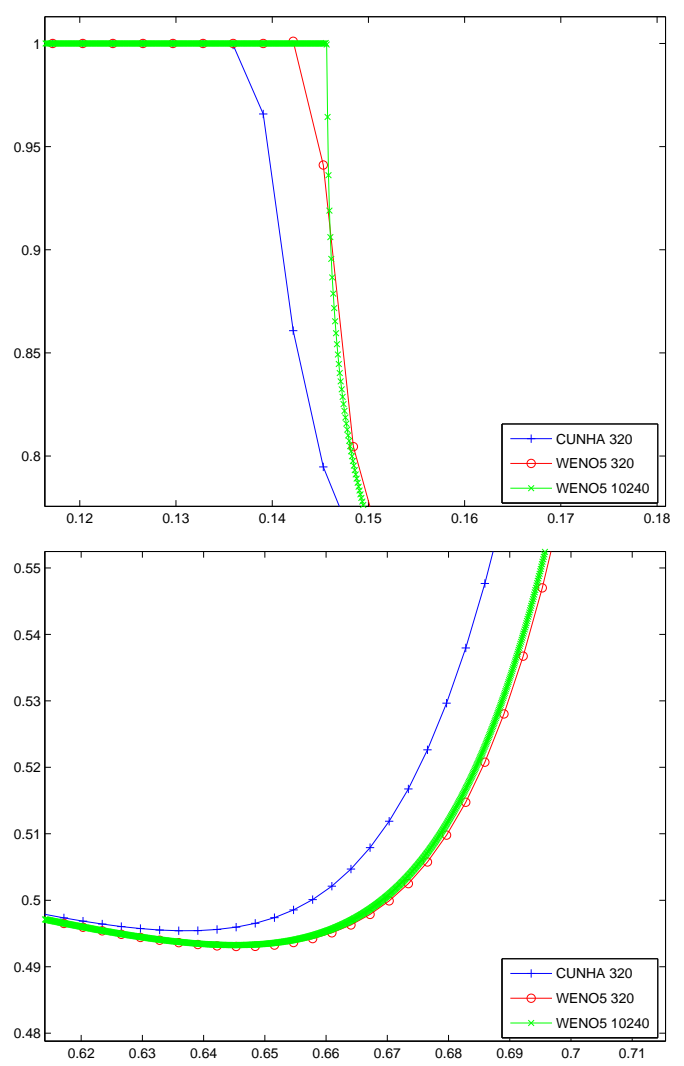

Fig. 9 Enlarged views of the approximated solutions shown in Figure 8.

In addition, in the IMEX-WENO scheme, we have to take into account that the running times depend on the value of the tolerance parameter $(\mathrm{Tol})$ taken in the Fixed Point Iteration. To determine which is the appropriate value we must use, we have tested how the error varies with different values of Tol. Figure 11 shows that the mean error is roughly constant when $T o l<10^{-3}$. In our simulations, we have set the value of the tolerance as Tol $=10^{-6}$.

\subsection{The role of the definition of $\alpha$}

Finally, we show what happens if we take the wrong definition of $\alpha$ in the WENO reconstruction of the numerical flux function.

In Figure 12, we can see the numerical solution given by the IMEX-WENO scheme with the definition of $\alpha$ given in (8). This means that for each interval $\left[s_{j}, s_{j+1}\right]$, we take

$$
\alpha_{j+1 / 2}=\max \left\{\left|F^{\prime}\left(s_{j}\right)\right|,\left|F^{\prime}\left(s_{j+1}\right)\right|\right\}
$$

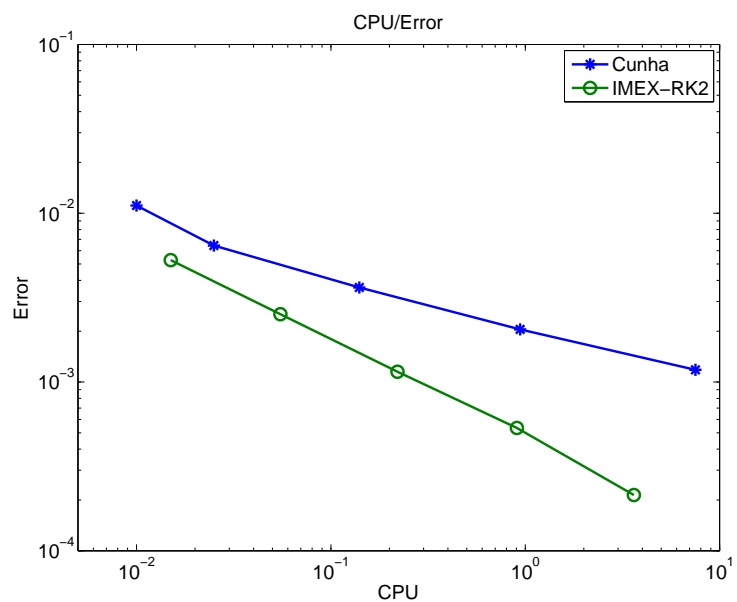

Fig. 10 Comparison between the efficiencies of the IMEXWENO scheme and the Godunov type scheme used in [5]. Both axes have logarithmic scaling.

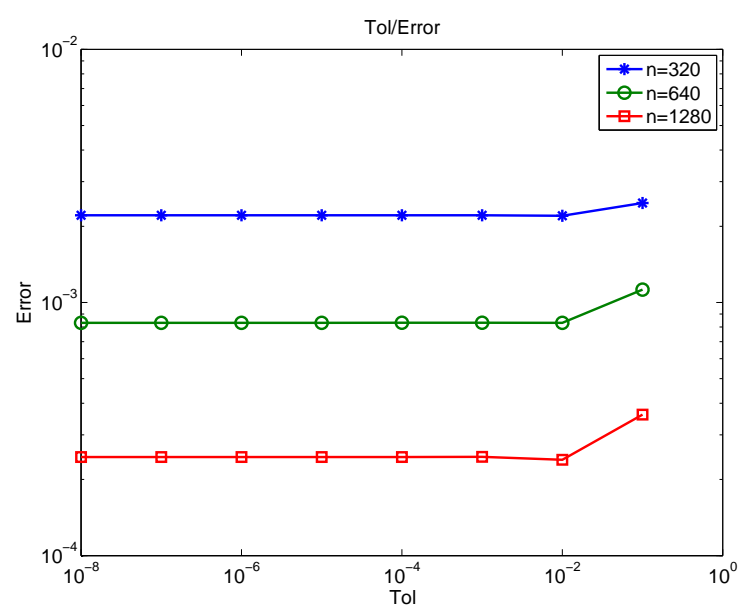

Fig. 11 Evolution of the mean errors as a function of the tolerance parameter $\mathrm{Tol}$ for different number of points.

The right shock shows a nonphysical oscillation due to the fact that the numerical viscosity $\alpha$ has a value which is smaller than necessary.

Figure 13 shows the same area of the numerical solution, but with the correct definition of the numerical viscosity $\alpha$ given in (9). This definition takes into account that any inflection point $S_{1}, S_{2}$ may be inside the interval $\left[s_{j}, s_{j+1}\right]$,

$$
\alpha_{j+1 / 2}=\max \left\{\left|F^{\prime}(s)\right|, s_{j} \leq s \leq s_{j+1}\right\}
$$



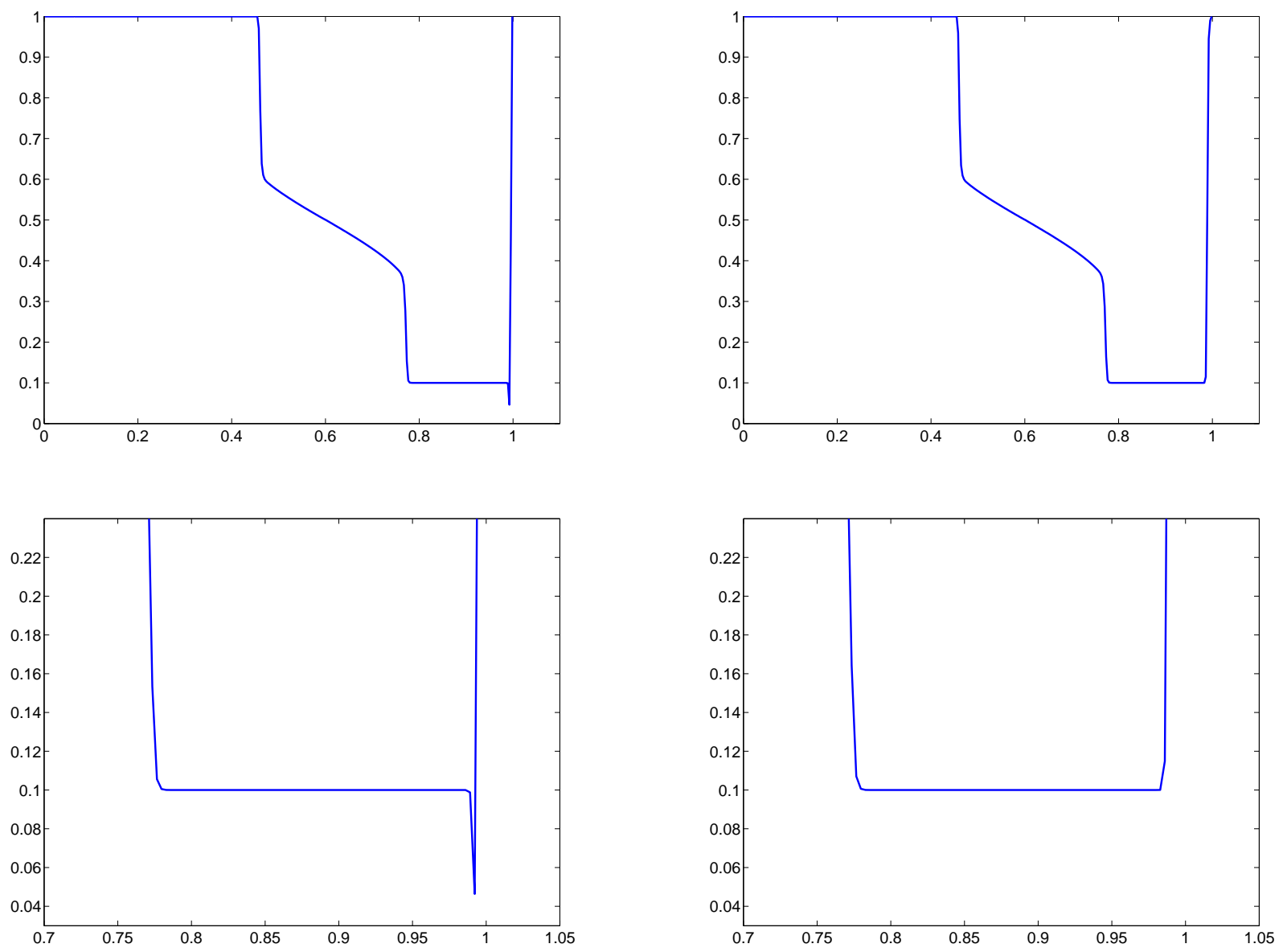

Fig. 12 Oscillations in the numerical solution with the wrong definition of $\alpha$ (8). Left: numerical solution for $T=0.5$ with $\beta=0.1, N=0, h=1 / 320$ and $k / h=0.1$. Right: zoom of the right shock area.

In particular, if $S_{i} \in\left[s_{j}, s_{j+1}\right]$ then, $\alpha_{j+1 / 2}=\left|F^{\prime}\left(S_{i}\right)\right|$, for $i=1,2$.

In this case the oscillation does not appear. Therefore, a right choice of the numerical viscosity parameter is a key point in the correct behavior of the numerical solutions obtained with a WENO scheme.

\section{Conclusions}

The advantages of using a shock-capturing scheme for the numerical simulation of hyperbolic or parabolic equations such as (1) was pointed out in [5], where the first order Godunov numerical flux function was combined with a second order discretization of the parabolic term. In this paper, we have shown that the use of a modern

high-order, high-resolution WENO numerical flux function can lead to a considerable gain in accuracy and efficiency in the numerical simulations, both for the $N=0$ and $N \neq 0$ cases.

For $N=0$ the proposed scheme (13) has been tested on some initial-boundary value problems for which the exact solution can be computed using geometric arguments.

For the $N \neq 0$ case (17), it is seen that the numerical approximations converge to a solution with the expected properties. The results have been compared to those obtained with the numerical schemes proposed in [5] and, in all cases, the high resolution simulations are more accurate. In addition, this gain in accuracy leads to a gain in efficiency, since the quality of the solution is better on coarser meshes. 
As we have seen, the implicit strategy (IMEX) in the discretization of the parabolic term, for $N \neq 0$, has less stringent requirements for stability. This allows larger time steps and a gain in computation times. In addition, the use of a second order Runge-Kutta scheme improves the accuracy of the solution.

The combination of both advantages leads to an improvement in efficiency that makes the IMEX-WENO scheme outperform the Godunov scheme.

The numerical results shown in this paper clearly suggest that the use of a high-order/high resolution shock capturing scheme for the discretization of the buoyancy terms and an implicit-explicit strategy for the time discretization can be a powerful numerical tool, which can be easily adapted to explore other scenarios of engineering interest, such as

-To study of hysteresis effects.

-To study three, or more, phase flow problems, with the vertical equilibrium assumption.

-Non-homogeneous porous media.

-To investigate the effect of the capillary pressure in a different way to that of Brooks-Corey models.

We conclude that the strong points of the IMEX-WENO scheme proposed here are:

-The IMEX strategy to handle the capillary term by an implicit discretization leads to a noticeable increase in resolution power and efficiency.

-It gives accurate numerical solutions for any value of the capillary number $N$,

-It reproduces properly the structure of shocks and rarefactions of the exact solution (Godunov scheme does not, see Figure 7). It can not give unphysical solutions.

-The use of a correct definition of the numerical viscosity is crucial. Not being careful at this point can lead to solutions affected by numerical instabilities.

Acknowledgments: The authors acknowledge support from the Spanish MINECO through the project MTM-2011-22741.

\section{Appendix A}

We perform a linearized stability analysis for the equation (1):

$$
\frac{\partial s}{\partial t}+\frac{\partial}{\partial x} F(s)+N \frac{\partial}{\partial x}\left(F(s) \frac{\partial P_{c}(s)}{\partial x}\right)=0,
$$

The linearized equation for a small perturbation $u$ about a constant state $s^{(0)}$ is obtained by substituting $s=s^{(0)}+u$ into (1) and neglecting quadratic terms in $u$. This yields the following linearized version of (1):

$$
\frac{\partial u}{\partial t}+a \frac{\partial u}{\partial x}=b \frac{\partial^{2} u}{\partial x^{2}},
$$

where $a=F^{\prime}\left(s^{(0)}\right)$ and $b=-N F\left(s^{(0)}\right) P_{c}^{\prime}\left(s^{(0)}\right)$.

\section{Euler explicit scheme}

We perform the von Neumann stability analysis for (40) with $a, b>0$ and the scheme:

$$
u_{j}^{n+1}=u_{j}^{n}-\alpha\left(u_{j}^{n}-u_{j-1}^{n}\right)+\beta\left(u_{j+1}^{n}-2 u_{j}^{n}+u_{j-1}^{n}\right),
$$

where $\alpha=a k / h$ and $\beta=b k / h^{2}$.

Substituting $u_{j}^{n}=e^{\theta i j}$, for $i=\sqrt{-1}$ and suitable $\theta$, we get:

$$
u_{j}^{n+1}=e^{\theta i j} A(\theta),
$$

where

$$
A(\theta)=1+(\alpha+2 \beta)(\cos \theta-1)-i \alpha \sin \theta
$$

so that $|A|^{2} \leq 1, \forall \theta$, is equivalent to

$$
\begin{aligned}
2(\alpha+2 \beta)- & (\alpha+2 \beta)^{2}-\alpha^{2}+ \\
& +\left((\alpha+2 \beta)^{2}-\alpha^{2}\right) \cos \theta \geq 0, \quad \forall \theta,
\end{aligned}
$$

which is equivalent to:

$$
2(\alpha+2 \beta)(1-\alpha-2 \beta) \geq 0
$$

That is, $\alpha+2 \beta \leq 1$ or, equivalently:

$$
\frac{a k}{h}+\frac{2 b k}{h^{2}} \leq 1
$$

\section{Euler implicit-explicit scheme}

We perform the von Neumann stability analysis for (40) with $a, b>0$ and the scheme:

$$
u_{j}^{n+1}=u_{j}^{n}-\alpha\left(u_{j}^{n}-u_{j-1}^{n}\right)+\beta\left(u_{j+1}^{n+1}-2 u_{j}^{n+1}+u_{j-1}^{n+1}\right),
$$

where $\alpha=a k / h$ and $\beta=b k / h^{2}$.

Substituting $u_{j}^{n}=e^{\theta i j}, i=\sqrt{-1}$ and suitable $\theta$, and setting $u_{j}^{n+1}=A(\theta) e^{\theta i j}$ we get:

$$
A(\theta)=1-\alpha(1-\cos \theta+i \sin \theta)+2 \beta A(\theta)(\cos \theta-1),
$$


that is:

$$
A(\theta)=\frac{1-\alpha(1-\cos \theta)-i \alpha \sin \theta}{1+2 \beta(1-\cos \theta)},
$$

so that $|A|^{2} \leq 1, \forall \theta$, is equivalent to

$$
\alpha-\alpha^{2}+2 \beta^{2}+2 \beta-2 \beta^{2} \cos \theta \geq 0, \forall \theta,
$$

which is equivalent to $\alpha-\alpha^{2}+2 \beta \geq 0$.

So, the necessary and sufficient condition for stability $\forall \beta>0$ is $\alpha<1$ or, in terms of parameters $a, b$ defined above is:

$$
\frac{a k}{h} \leq 1
$$

\section{References}

[1] J. Aarnes, V. Kippe, K. A. Lie, A. B. Rustad, Modelling of multiscale structures in flow simulations for petroleum reservoirs, in: G. Hasle, K.A. Lie (Eds.), Geometric modeling, numerical simulation and optimization: Applied mathematics at SINTEF, Springer-Verlag, (2007).

[2] M. A. Christie, Upscaling of reservoir simulation, J. Pet. Technol, 1004-1010 (1996).

[3] J. Bear, V. Ryzhik, On the displacement of NAPL lenses and plumes in a phreatic aquifer, Transp. Porous Media, 33, 227 255 (1998).

[4] E. Mouche, M. Hayek, C. Mügler, Upscaling of $\mathrm{CO}_{2}$ vertical migration through a periodic layered porous medium: The capillary-free and capillary-dominant cases, Adv. Water Resour, 33, 1164-1175 (2010).

[5] M. C. C. Cunha, M. M. Santos, J. E. Bonet, BuckleyLeverett mathematical and numerical models describing vertical equilibrium process in porous media, Int. J. Eng. Sci., 42, 1289-1303 (2004).

[6] H. Darcy, Les fontaines publiques de la ville de Dijon, Dalmont, Paris, (1856).

[7] R. H. Brooks, A. T. Corey, Properties of porous media affecting fluid flow, J. Irrig. Drain. Div. Am. Soc. Civ. Eng., 92, 61-88 (1966).

[8] M. C. Leverett, Capillary behaviour in porous solids, Trans. AIME Petr. Eng. Div., 142, 152-169 (1941).

[9] R. LeVeque, Numerical Methods for Conservation laws, Birkhauser-Verlag, (1990).

[10] I. S. Pop et al, Horizontal redistribution of fluids in a porous medium: The role of interfacial area in modeling hysteresis, Adv. Water Resour, 32, 383-390 (2009).

[11] X. Liu, S. Osher, T. Chan, Weighted essentially nonoscillatory schemes, Journal of Computational Physics, 115, 200-212 (1994).

[12] G. S. Jiang and C. W. Shu. Efficient implementation of weighted ENO schemes, J. Comput. Phys., 126, 202-228 (1996).

[13] B. Cockburn, C. Johnson, C. W. Shu, E. Tadmor, Essentially non-oscillatory and weighted essentially non-oscillatory schemes for hyperbolic conservation laws, in: A. Quarteroni (Ed.), Advance Numerical Approximations of Nonlinear Hyperbolic Equations, Lecture Notes in Mathematics, Springer, Berlin, 1697, 325-432 (1998).
[14] A. Harten et al, Uniformly high order essentially nonoscillatory schemes III, J. Comput. Phys., 71, 231-303 (1987).

[15] C. W. Shu. High order weighted essentially nonoscillatory schemes for convection dominated problems. SIAM Rev., 51, 82-126 (2009).

[16] C. W. Shu, S. Osher, Efficient implementation of essentially non-oscillatory shock-capturing schemes II, J. Comput. Phys., 83, 32-78 (1989).

[17] U. M. Ascher, S. J. Ruuth and R. Spiteri, Implicit-explicit Runge-Kutta methods for time dependent partial differential equations, Applied Numerical Mathematics, 25, 151-167 (1997).

[18] Bürger, R. and Fjelde, K. K. and Höfler, K. and Karlsen, K. Hvistendahl, Central difference solutions of the kinematic model of settling of polydisperse suspensions and threedimensional particle-scale simulations, J. Engineering. Math., 41, 167-187 (2001).

[19] R. Bürger, R. Donat, P. Mulet and C. A. Vega, On the implementation of WENO schemes for a class of polydisperse sedimentation models, J. Comput. Phys., 230, 2322-2344 (2011). 


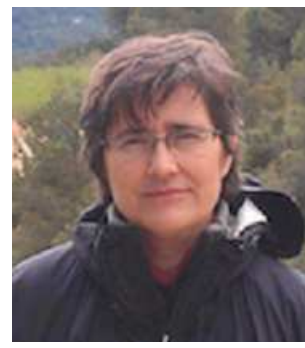

Rosa M. Donat obtained her M.Sc. in 1987 and her Ph.D. in 1990, both in mathematics from the University of California, Los Angeles, USA. She is currently full professor at the applied mathematics department of the University of Valencia, Spain. Her research interests include: high resolution methods for hyperbolic systems of partial differential equations, Harten multiresolution and image processing. She has published more than 50 research articles in reputed international journals of mathematical and engineering sciences.

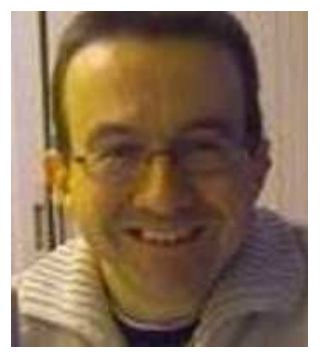

\section{Francisco Guerrero} received the $\mathrm{PhD}$ degree in Theoretical Physics at the University of Valencia in 1998. He is currently assistant professor at the applied mathematics department of the University of Valencia. His research interests are in the areas of mathematical methods for dynamic systems, transport in porous media and numerical methods for partial differential equations. He has published research articles in reputed international journals of mathematical and physical sciences.

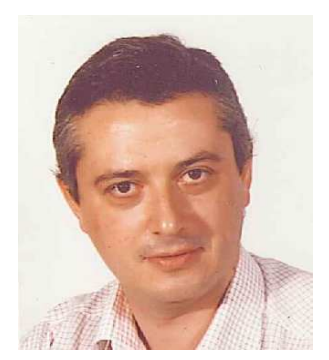

Pep Mulet obtained his M.Sc. in 1989 and his Ph.D. in 1992, both in mathematics from the University of Valencia, Spain. $\mathrm{He}$ is currently associate professor at the applied mathematics department of the University of Valencia. He is now head of this department. His research interests include image processing, adaptive scientific computing and numerical analysis of partial differential equations. He has published more than 30 research articles in reputed international journals of mathematical and engineering sciences. 